\title{
Obtaining of Thin Ophthalmic Polymer Films
}

\author{
Yunusov Kh.E.*, Shukurov A.I., Sarymsakov A.A. and Rashidova S.Sh.
}

Institute of polymer chemistry and physics, Uzbekistan Academy of Sciences, Uzbekistan

*Corresponding author: Yunusov Kh E, Institute of polymer chemistry and physics, Uzbekistan Academy of Sciences, Uzbekistan

Submission: February 06, 2019; Published: February 22, 2019

\begin{abstract}
In this paper possibility of obtaining thin ophthalmic polymer drug films (OPDF) and their structure and physico-chemical properties were studied. By using atomic force microscopy and UV-spectroscopy analysis the shapes and sizes of nanoparticles of "SelAgrip" substance in the structure of sodium carboxymethylcellulose films were determined.
\end{abstract}

\section{Introduction}

Thin films, developed and characterized with Nanotechnology methods, are commonly used as biomaterials because of their unique interfacial and mechanical properties. Application of thin films in medical specialties such as cardiology [1], orthopedics [2] and urology [3] leads to new approaches to therapeutic challenges. Obstacles related to safety and cost effectiveness of nanotechnology research remain, however those are not insurmountable and thin films are expected to contribute significantly to biomedical research. Primary aim of this work is to discuss applications of thin films in diagnosis, monitoring and treatment of ophthalmic diseases.

The pioneering group of polymers used as components of ophthalmic preformed hydrogelsisthefamilyofcellulosicderivatives. Because pure cellulose is not water soluble due to its relatively high crystallinity, cellulosic derivatives have been used for a long time as viscosifiers in collyria. Methylcellulose (MC) was first introduced in ophthalmic formulations in the 1940 s as a mean of decreasing their fluidity. Since then, cellulosic polymers have been extensively studied in human, as well as in veterinary medicine, for ocular administration. The cellulosic derivatives most commonly used in ophthalmology are: Methylcellulose, Hydroxyethylcellulose (HEC), Hydroxypropycellulose (HPC), Hydroxypropylmethylcellulose (HPMC) and Sodium carboxymethylcellulose (Na-CMC).

Ophthalmic films is the one of the new dosage forms intended for the prevention and treatment of eye viruses diseases of various etiologies. The goal of this research is development of ophthalmic films based on water-soluble purified Na-CMC with degree of substitution (DS)=0.75-0.85 and degree of polymerization (DP) $=350-850$, contained antiviral drug "SelAgrip" which approved for use in medical practice as antiviruses medicine.

At the first stage of investigation ophthalmic films from aqueous solutions of Na-CMC of different DP and DP were obtained and their physico-chemical and physico-mechanical properties were studied. Bio-soluble antiviral ophthalmic polymeric eye films were obtained by dispersing aqueous solutions of biologic active substance of "SelAgrip" in aqueous solutions of Na-CMC. The ophthalmic films was obtained from $2 \%$ of aqueous Na-CMC solutions on the surface glass plates. After the solvent-water was removed, ophthalmic films were formed (Figure 1A,B).

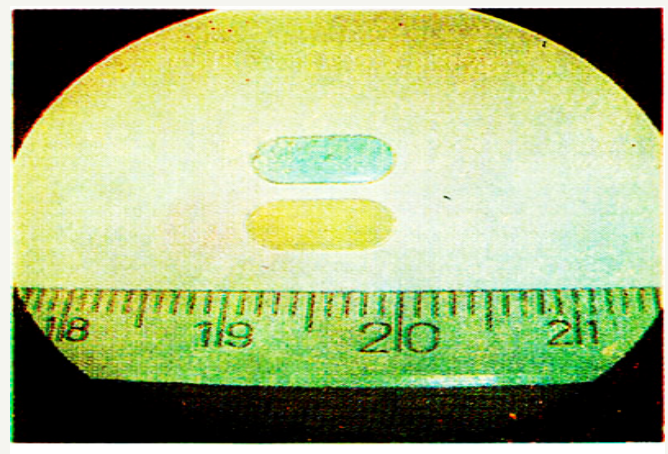

A

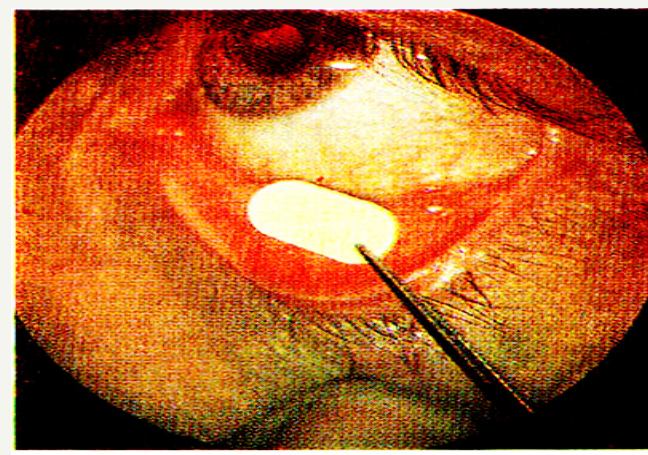

B

Figure 1A,B: Ophthalmic drug film with the size $8 \mathrm{x} 4 \mathrm{~mm}$ and its application in ophthalmology. 
The use of various DS and DP as polymer matrix of Na-CMC contributed to the regulation of the periods of swelling and dissolution of the ophthalmic drug film in the tear fluid during 20-90 minutes. It is established that when the concentration of substance in the composition of the polymer matrix varies from 6 to $20 \%$, the ophthalmic drug films exhibit low toxicity and high specific antiviral activity. Ophthalmic drug films (ODF) are oval- shaped plates consisting of a biologically soluble polymer and a drug substance compatible with eye tissues.

It can be seen from Figure 2, nanoparticles of "SelAgrip" substance spherical form with sizes $30 \pm 5 \mathrm{~nm}$ with a fairly high uniformity of nanoparticle size distribution are observed on the surface of the films.
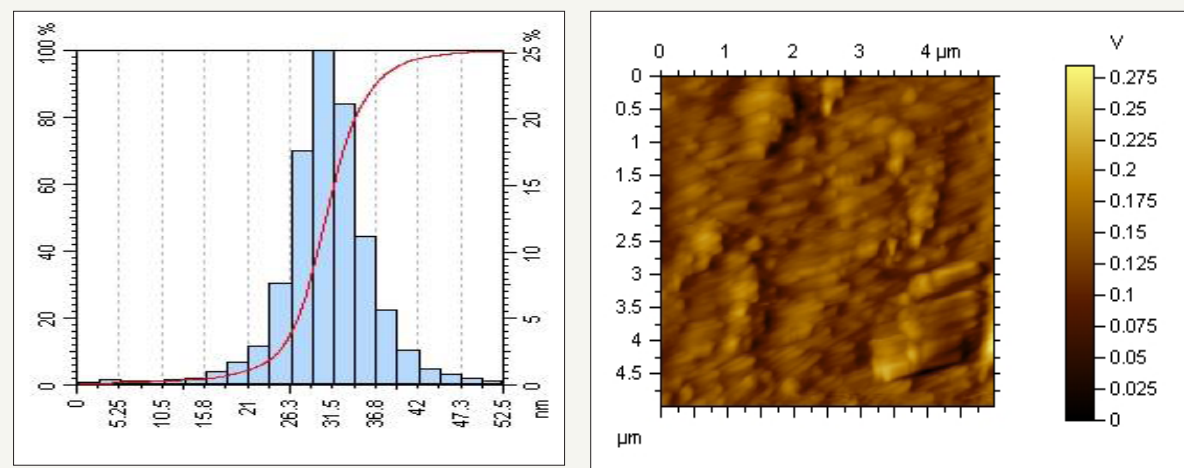

Figure 2: Electron micrographs of the ophthalmic drug films and the uniform distribution of the substance nanoparticles in the film structure.

Ophthalmic drug films allowed to:

a) to carry out accurate, controlled dosing of drugs;

b) to ensure the prolongation of their action as a result of the slow, gradual dissolution of the film in the tear fluid;

c) reduce the number of injected drugs several times;

d) to increase the therapeutic concentration of medicinal substances in the tissues of the eye;

e) reduce the course of treatment by 2-3 times;

f) carry out treatment in such conditions when the use of other dosage forms is difficult or impossible;

g) completely dissolved in the conjunctival sac.

\section{Conclusion}

On the base of the results of experimental investigations established that, by changing parameter reaction process should be obtain prolonged form of ophthalmic drug films based on sodium carboxymethylcellulose and biologically active substance "SelAgrip". Application of prolonged form of ophthalmic drug films for the treatment of various ophthalmic diseases, improves the efficiency and reduces the negative impact of drugs on healthy organs.

The work was performed as part of the innovation project No. IZ-20170920233 "Development of technology and mastering the production of nanostructured, bio-soluble, antiviral eye medicinal films "GlazAvir"" Ministry of Innovative Development of the Republic of Uzbekistan.

\section{References}

1. Huygens H (1951) Three memoir on mechanics. M USSR Academy of Sciences, Russia.

2. Appell P, Lacour E (1897) Principes de la Theorie des Fonctions Elliptiques et Applications. Gauthier-Villars, Paris, France.

3. Appel PA (1960) Theoretical mechanics.

4. Adlaj S. An eloquent formula for the perimeter of an ellipse. Notices of the AMS 59(8): 1096-1097.
Creative Commons Attribution 4.0 International License

For possible submissions Click Here
Submit Article

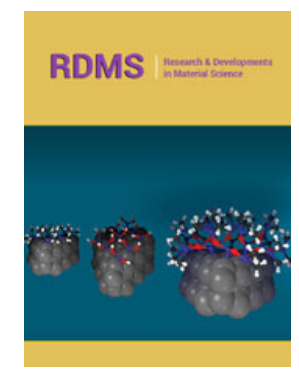

Research \& Development in Material Science

\section{Benefits of Publishing with us}

- High-level peer review and editorial services

- Freely accessible online immediately upon publication

- Authors retain the copyright to their work

- Licensing it under a Creative Commons license

- Visibility through different online platforms 